PROCEEDINGS OF THE

AMERICAN MATHEMATICAL SOCIETY

Volume 124, Number 12, December 1996, Pages 3689-3698

S 0002-9939(96)03422-3

\title{
A COCYCLE THEOREM WITH AN APPLICATION TO ROSENTHAL SETS
}

\author{
PETER SCHWARTZ
}

(Communicated by J. Marshall Ash)

\begin{abstract}
For certain Markov operators $T$ we show that bounded cocycles with respect to $T$ are coboundaries. This result is applied to show that certain translation invariant subspaces of functions on the unit circle have unexpected regularity properties.
\end{abstract}

\section{INTRODUCTION}

Gottschalk and Hedlund [GH] published the following theorem: Let $Y$ be a compact topological space and $\theta$ a homeomorphism of $Y$. Suppose that for all $y \in Y$, $\left\{\theta^{n}(y): n \in \mathbf{Z}\right\}$ is dense in $Y$. Suppose there is a continuous function $F$ and a positive number $K$, such that for all $N,\left\|\sum_{n=0}^{N} F \circ \theta^{n}\right\|_{\infty} \leq K$. Then there exists a continuous function $g$ such that $g-g \circ \theta=F$. In more abstract language, one says that if $F$ is a bounded cocycle, then $F$ is a coboundary. Using different methods, this paper establishes a similar theorem for the sums $\sum_{n=0}^{N} T^{n} F$, where $T$ is a uniquely ergodic Markov operator whose invariant measure has full support. In section 2 , the cocycle theorem is applied to show that finitely generated multiplicative semigroups in the integers are Rosenthal sets. In the concluding theorem arguments from section 1 are modified to produce a proof that the product of a finitely generated multiplicative semigroup and a Sidon set is a Rosenthal set. Finally, some previous examples [Ro, PS1, PS2] of Rosenthal sets are realized as special cases of the results above.

Notation and Definitions. Let $X$ be a compact metric space. We denote the continuous complex-valued functions on $X$ by $\mathbf{C}(X)$. An operator $T: \mathbf{C}(X) \rightarrow$ $\mathbf{C}(X)$ that satisfies (1) $T f \geq 0$ if $f \geq 0$ and (2) $T 1=1$ is called a Markov operator. Standard compactness arguments show there are always $T$-invariant probability measures on $X$. We say that $T$ is uniquely ergodic if there exists a unique $T$ invariant Borel probability measure on $X$. A measure $m$ has full support if $m(O)>$ 0 for all open sets $O$. Finally, given $x \in X$ and $\varepsilon>0$ we denote the ball of radius $\varepsilon$ around $x$ by $B(x, \varepsilon)$.

1.

1.1. Theorem. Let $X$ be a compact metric space. Let $T: \mathbf{C}(X) \rightarrow \mathbf{C}(X)$ be a Markov operator. Let $F \in \mathbf{C}(X)$. Suppose

Received by the editors February 17, 1994 and, in revised form, March 25, 1995. 1991 Mathematics Subject Classification. Primary 47A99, 42A16, 42A55.

(C)1996 American Mathematical Society 
(1) There exists a constant $K<\infty$ such that for all $N,\left\|\sum_{n=0}^{N} T^{n} F\right\|_{\infty} \leq K$.

(2) $T$ is uniquely ergodic and the unique T-invariant Borel probability measure has full support.

Then there exists a continuous function $f$ such that $F=f-T f$.

Proof. Denote the $T$-invariant probability measure by $m$. We require the following lemmas, some of which are standard results. In the latter case we sometimes give a reference rather than a proof. We begin with a definition.

1.2. Definition. Let $p$ be a real-valued, bounded function on $X$. We define, $\bar{p}$ : $K \rightarrow \mathbf{R}$, the upper-semicontinuous cover of $p$, by $\bar{p}(x)=\inf \{q(x): q$ is continuous and $q \geq p\} \cdot \bar{p}$ is the least upper-semicontinuous function greater than $p$.

1.3. Lemma. Let $T: \mathbf{C}(X) \rightarrow \mathbf{C}(X)$ be a Markov operator on a compact metric space. Then $T$ may be extended to an operator from the set of bounded Borel functions to itself. Furthermore, under this extension the image of an uppersemicontinuous function is upper-semicontinuous. And finally, $T \bar{p} \geq \overline{T p}$ for all bounded Borel functions $p$.

Proof. The extension of $T$ to bounded Borel functions is exhibited in [Fo]. In [Me] it is shown that the image of an upper-semicontinuous function is again uppersemicontinuous. If $p$ is bounded, Borel, then $T \bar{p} \geq T p$ by the positivity of $T$, and $T \bar{p}$ is upper-semicontinuous. Therefore $T \bar{p}$ is also greater than the least uppersemicontinuous function greater than $T p$. That is, $T \bar{p} \geq \overline{T p}$.

1.4. Definition. Given a compact metric space $X$ and a Borel probability measure $m$ on $X$, we define $\mathcal{R}_{m}$, the Riemann integrable functions with respect to $m$, by the condition: $\mathcal{R}_{m}=\left\{h\right.$ : for all $\varepsilon>0$ there exist continuous functions $h_{1}$ and $h_{2}$ such that $h_{1} \geq h \geq h_{2}$ and $\left.\int h_{1}-h_{2} d m \leq \varepsilon\right\}$.

1.5. Lemma. Let $X$ be a compact metric space and $m$ a Borel probability measure. $A$ bounded function $h$ is in $\mathcal{R}_{m}$ if and only if the points of continuity of $h$ form a set of full m-measure.

Proof. If $X$ is an interval in the real line and $m$ is Lebesgue measure on the line, then the assertion is part of the standard development of the theory of Riemann integrable functions. See, for example, [St, page 269]. The proof for general $X$ and $m$ is similar. We omit the details.

1.6. Lemma. Let $m$ be a probability measure with full support on a compact metric space. If a bounded complex-valued function $h$ is continuous when restricted to a set $A$, for some set $A$ of $m$-measure 1 , then $h$ is $m$-equivalent to a function that is Riemann integrable with respect to $\mathrm{m}$.

Proof. A function is Riemann integrable if and only if its real and imaginary parts are Riemann integrable. Therefore without loss of generality $h$ is real-valued. Let

$$
\tilde{h}(x)=\limsup _{\varepsilon \rightarrow 0^{+}} \frac{1}{m\{B(x, \varepsilon)\}} \int_{B(x, \varepsilon)} h(t) d m(t) .
$$

Then if $x \in A$,

$$
\begin{aligned}
\tilde{h}(x)-h(x) & =\limsup _{\varepsilon \rightarrow 0^{+}} \frac{1}{m\{B(x, \varepsilon)\}} \int_{B(x, \varepsilon)}(h(t)-h(x)) d m(t) \\
& =\limsup _{\varepsilon \rightarrow 0^{+}} \frac{1}{m\{B(x, \varepsilon)\}} \int_{B(x, \varepsilon)}\left(h(t) \mathbf{1}_{A}(t)-h(x)\right) d m(t) .
\end{aligned}
$$


This last quantity equals zero since $h$ restricted to $A$ is continuous. On the other hand, $\tilde{h}(x)$ is continuous for all $x \in A$, as can be seen from the following argument. Fix $x \in A$. Given $\delta>0$ find $\eta>0$ such that $|h(y)-h(x)| \leq \delta$ if $y \in B(x, \eta)$ and $y \in A$. Let $y \in B\left(x, \frac{\eta}{2}\right)$. For $0<\varepsilon<\frac{\eta}{2}$, we have

$$
\frac{1}{m\{B(y, \varepsilon)\}} \int_{B(y, \varepsilon)}|h(x)-h(t)| \mathbf{1}_{A}(t) d m(t) \leq \delta,
$$

since the integrand is smaller than $\delta$ for every $t$. Thus

$$
\begin{aligned}
\mid \tilde{h}(y) & -\tilde{h}(x)|=| \tilde{h}(y)-h(x) \mid \\
& \leq \limsup _{\varepsilon^{+} \rightarrow 0} \frac{1}{m\{B(y, \varepsilon)\}} \int_{B(y, \varepsilon)}|h(x)-h(t)| \mathbf{1}_{A}(t) d m(t) \leq \delta .
\end{aligned}
$$

Thus the points of continuity of $\tilde{h}$ are of full $m$-measure, which implies by Lemma 1.5 that $\tilde{h} \in \mathcal{R}_{m}$.

1.7. Lemma. Let $X$ be a compact metric space. Let $T: \mathbf{C}(X) \rightarrow \mathbf{C}(X)$ be a uniquely ergodic Markov operator with invariant measure $m$.

(1) If $h \in \mathcal{R}_{m}$, then $A_{N}(h)=\frac{1}{N} \sum_{n=0}^{N} T^{n} h$ converges uniformly to $\int h d m$ as $N$ tends to infinity.

(2) If $g \in \mathbf{L}^{1}(m)$ and $T g=g$-a.e., then $g$ is m-a.e. constant.

Proof of (1). Without loss of generality $\int h d \mu=0$. For $h$ continuous, the assertion is included in Proposition 1.2 of [Kr, p. 178]. For $h$ merely in $\mathcal{R}_{m}$ we recall that given $\varepsilon>0$ there exist continuous functions $h_{1}$ and $h_{2}$ such that $h_{1} \leq h \leq h_{2}$ and $\int h_{2}-h_{1} d \mu \leq \varepsilon$. Now the positivity of $T$ implies that for all $N$

$$
A_{N}\left(h_{1}\right) \leq A_{N}(h) \leq A_{N}\left(h_{2}\right) .
$$

Since $h_{1}$ and $h_{2}$ are continuous for sufficiently large $N$ we have $-2 \varepsilon \leq \lim \sup A_{N}(h)$ $\leq 2 \varepsilon$ and $-2 \varepsilon \leq \liminf A_{N}(h) \leq 2 \varepsilon$. Since $\varepsilon$ is arbitrary $A_{N}(h)$ converges uniformly to 0 , which proves (1).

Proof of (2). Observe that $\int|T g| d m \leq \int T|g| d m \leq \int|g| d m$. Hence $T$ is an $\mathbf{L}^{1}(m)$ contraction. Now $\mathbf{C}(X)$ is dense in $\mathbf{L}^{1}(m)$ and a standard approximation argument shows that $\lim _{N \rightarrow \infty} A_{N}(g)=\int g d m$ in the norm of $\mathbf{L}^{1}(m)$ for all $g \in \mathbf{L}^{1}(m)$. This last statement implies that the only invariant functions are a.e. constant which is the assertion of (2).

1.8. Lemma. Let $m$ be a probability measure with full support on a compact metric space. Let $\left(h_{n}\right)$ be a Cauchy sequence of bounded functions in the uniform norm, such that for all $n, h_{n}$ is m-equivalent to a continuous function, then $h=\lim _{n} h_{n}$ is $m$-equivalent to a continuous function.

Proof. By hypothesis there exists for each $n$ a continuous function $g_{n}$ such that $g_{n}=h_{n} m$-a.e. Given $\varepsilon>0$ there exists $M$ such that for $j, k \geq M,\left|h_{j}-h_{k}\right| \leq \varepsilon$ except on a set of $m$-measure 0 . In addition, the continuity of $g_{k}$ and the hypothesis that $m$ is of full-support imply that for $j, k \geq M,\left|g_{j}-g_{k}\right| \leq \varepsilon$ everywhere. Thus $\left(g_{k}\right)$ is a Cauchy sequence in the uniform norm. Let $g=\lim _{k} g_{k} ; g$ is continuous. Choose $\varepsilon>0$. Except on a set of $m$-measure $0,|h-g| \leq\left|h-h_{j}\right|+\left|g-g_{j}\right|+\left|g_{j}-h_{j}\right| \leq \varepsilon$ if $j$ is large. Since $\varepsilon$ is arbitrary $h$ is $m$-equivalent to $g$. 
We now begin the proof of Theorem 1.1. The first step is to show that the functional equation, $F=f-T f \mu$-a.e. has a solution among the upper-semicontinuous functions. $F$ is a complex-valued function. However, since $T F$ is realvalued if $F$ is real-valued, each of the real and complex parts of $F$ is a bounded cocycle in their own right with respect to $T$. Therefore if the theorem holds for realvalued $F$, then the theorem holds for complex-valued $F$ as well. Hence we assume without loss of generality that $F$ is real-valued. We denote the unique $T$-invariant measure by $m$. We remark for later use that the hypothesis that $F$ is a bounded cocycle implies that $\int F d m=0$. Let $f(x)=\lim \sup _{N \geq 0} \sum_{n=0}^{N} T^{n} F(x)$. Then $f$ is bounded, Borel and

$$
T f \geq \underset{N \geq 0}{\limsup } \sum_{n=0}^{N} T^{n+1} F=\underset{N \geq 0}{\limsup } \sum_{n=0}^{N+1} T^{n} F-F=f-F .
$$

In addition

$$
T \bar{f} \geq \overline{T f} \geq \overline{f-F}=\bar{f}-F .
$$

The first inequality of (2) follows from Lemma 1.3; the second follows from equation (1); and the equality follows from the continuity of $F$. Furthermore, from equation (1), we see that $T f-f+F \geq 0$. Since, in addition, $\int(T f-f+F) d m=$ 0 , we infer that $T f-f+F=0 m$-a.e. Similarly, $T \bar{f}-\bar{f}+F=0 m$-a.e. Hence $T(f-\bar{f})=(f-\bar{f}) m$-a.e. Since $T$ is uniquely ergodic the only fixed points of $T$ are constants (Lemma 1.7) and thus $(f-\bar{f})$ is $\mu$-a.e. a constant, which implies that $f$ is $m$-equivalent to an upper-semicontinuous function. Let $G=-F$. Define $g(x)=\lim \sup _{N \geq 0} \sum_{n=0}^{N} T^{n} G$. The same reasoning as above shows that $g$ is equivalent to an upper-semicontinuous function. Applying (1) to $f$ and to $g$ and adding yields $T(f+g) \geq(f+g)$, which implies that $T(f+g)=$ $(f+g) m$-a.e. The unique ergodicity of $T$ implies that $f+g$ is $m$-a.e. a constant, which implies that $f$ is $m$-equivalent to a lower- as well as an upper-semicontinuous function. Hence there exists a set, $A$, of full $m$-measure such that $f$ restricted to $A$ is continuous. By Lemma 1.6, $f$ is $m$-equivalent to a Riemann integrable function $h \in \mathcal{R}_{m}$. Furthermore, we may without loss of generality choose $h$ with $\int h d m=0$. We now show that $h$ is $m$-equivalent to a continuous function. Since $F=h-T h$ $m$-a.e., we have for all $N$ and $m$-a.e.

$$
\frac{1}{N} \sum_{n=1}^{N} \sum_{j=0}^{n-1} T^{n} F=\frac{1}{N} \sum_{n=1}^{N} \sum_{j=0}^{n-1} T^{n}(h-T h)=h-\frac{1}{N} \sum_{n=1}^{N} T^{n} h .
$$

For each $N$, the right side of equation (3) is $m$-equivalent to a continuous function. By Lemma 1.7, $\frac{1}{N} \sum_{n=1}^{N} T^{n} h$ converges uniformly to 0 . Therefore $h$ is evidently the uniform limit of functions that each are equivalent to continuous functions, which implies by Lemma 1.7 that $h$ itself is equal almost everywhere to a continuous function. Let $\tilde{h} \equiv h$ with $\tilde{h} \in \mathbf{C}(X)$. Then $\tilde{h}-T \tilde{h} \in \mathbf{C}(X)$ and $\tilde{h}-T \tilde{h}=F m$-a.e. Since $F$ is also continuous the equation holds everywhere.

1.9. Remark. Let $X$ be a compact metric space. Let $T: \mathbf{C}(X) \rightarrow \mathbf{C}(X)$ be a Markov operator. Let $F \in \mathbf{C}(X)$. Suppose

(1) $T$ is uniquely ergodic and the unique $T$-invariant Borel probability measure has full support. 
(2) There exists a constant $K<\infty$ such that for all $N$, the essential supremum (with respect to the $T$-invariant measure) of $\left\|\sum_{n=0}^{N} T^{n} F\right\|_{\infty} \leq K$. Then the same argument shows that there exists a continuous function $f$ such that $F=f-T f$.

1.10. Remark. With few changes the proof of Theorem 1.1 can be adapted to give another proof of the Gottschalk-Hedlund theorem. For general operators Lin and Sine [LS] have observed that the left side of equation (3) converges in norm if and only if $F$ is a coboundary.

2.

We now apply the cocycle theorem to the study of translation invariant subspaces of $2 \pi$-periodic functions.

Notation and Definitions. Let $\mathbf{T}$ denote the circle-group. In what follows $\mathbf{T}$ will be modeled by the interval $[0,1)$ with addition mod 1 . Lebesgue measure will be denoted by $m . \mathbf{L}^{\infty}(\mathbf{T})$ and $\mathbf{L}^{q}(\mathbf{T})$ will denote respectively the Lebesgue measurable bounded and $q$ th-power integrable (equivalence class of) complex-valued functions. If $f=g$ a.e., then we sometimes write $f \equiv g . \mathbf{C}(\mathbf{T})$ will denote the continuous complex-valued functions. Similarly $\mathbf{C}_{0}(\mathbf{T})$ will denote the functions in $\mathbf{C}(\mathbf{T})$ with mean 0. $\mathbf{A}(\mathbf{T})$ will denote functions in $\mathbf{L}^{1}(\mathbf{T})$ with absolutely summable Fourier coefficients. Given a function $h \in \mathbf{L}^{1}(\mathbf{T})$, define the spectrum of $h$ to be the set of $k$ such that $\hat{h}(k) \neq 0$. Given a function space $H \subset \mathbf{L}^{\infty}(\mathbf{T})$ and a subset of integers $S, H_{S}$ will denote the set of $h \in H$ with spectrum in $S$. A Rosenthal set, $R$, in the integers (or the dual of any compact abelian group) is defined by the property that every bounded function with spectrum in $R$ is equivalent to a continuous function. Generally this equivalence is written as $\mathbf{L}_{R}^{\infty}(\mathbf{T})=\mathbf{C}_{R}(\mathbf{T})$. A Sidon set, $S$, in the integers (or the dual of any compact abelian group) is defined by the property that $\mathbf{L}_{S}^{\infty}(\mathbf{T})=\mathbf{A}_{S}(\mathbf{T}) . \mathbf{M}(\mathbf{T})$ is the set of Borel measures, and $\mu \in \mathbf{M}(\mathbf{T})$ is said to be adapted if the support of $\mu$ is not contained in a proper closed subgroup. For $z \in[0,1), \delta_{z}$ denotes the Dirac mass at $z$. Given two sets of integers, $M$ and $N$, define the product $M N=\{k: k=m n$ for some $m \in M$ and $n \in N\}$ and the sum $M+N=\{k: k=m+n$ for some $m \in M$ and $n \in N\}$. We say that a set of integers $M$ is symmetric if $M=-M$.

2.1. Theorem. Let $S \subset \mathbf{Z}$ be a finitely generated multiplicative semigroup. Then $S$ is a Rosenthal set. That is, $\mathbf{L}_{S}^{\infty}=\mathbf{C}_{S}$.

We require a lemma verifying that Theorem 1.1 applies.

2.2. Lemma. If $\mu \in \mathbf{M}(\mathbf{T})$ is an adapted probability measure, then the Markov operator $T: \mathbf{C}(\mathbf{T}) \rightarrow \mathbf{C}(\mathbf{T})$ given by $T h=\mu * h$ is uniquely ergodic, and the unique invariant measure is Lebesgue measure.

Proof. Let $\nu$ be a $T$-invariant probability measure. That is, $\int h d \nu=\int \mu * h d \nu$ for all $h \in \mathbf{C}(\mathbf{T})$. This implies that $\mu * \nu=\nu$. Furthermore, the hypothesis that $\mu$ is adapted implies that $\hat{\mu}(k) \neq 1$ for all $k \neq 0$. Thus $\hat{\nu}(k)=0$ for all $k \neq 0$, and since $\nu$ is a probability measure, $\hat{\nu}(0)=1$. We have shown that Lebesgue measure is the only $T$-invariant measure.

Proof of Theorem 2.1. Fix a sequence $\left(c_{j}\right)$ satisfying

(1) $c_{j}>0$, 
(2) $\sum_{j=0}^{\infty} c_{j}=1$

(3) for all $m>0, \sum_{j=m+1}^{\infty} c_{j} \leq \frac{1}{m^{2}}$.

By hypothesis there are primes $p_{1}, p_{2}, \ldots, p_{N}$ that generate $S$. For $i=1,2, \ldots, N$ let $\mu_{i}=\sum_{j=1}^{\infty} c_{j} \delta_{p_{i}^{-j}}$. Each $\mu_{i}$ is an adapted probability measure that has the property that if

$$
p_{i}^{l} \mid k, \text { then }\left|1-\hat{\mu}_{i}(k)\right| \leq \frac{\sqrt{2}}{l^{2}} .
$$

For $n=1,2, \ldots, N$ let

$$
F_{n}=\prod_{l=1}^{n}\left(\delta_{0}-\mu_{l}\right) \in \mathbf{M}(\mathbf{T})
$$

where the product symbol denotes convolution. Then if $g \in \mathbf{L}_{S}^{\infty}, F_{N} * g \in \mathbf{A}(\mathbf{T})$. Indeed

$$
\begin{aligned}
\left\|F_{N} * g\right\|_{\mathbf{A}} & \leq\|g\|_{\infty} \sum_{d_{i} \geq 0}\left|\prod_{l=1}^{N}\left(1-\hat{\mu}_{l}\left( \pm p_{1}^{d_{1}} \cdots p_{n}^{d_{n}}\right)\right)\right| \\
& \leq 2\|g\|_{\infty} \sum_{d_{i} \geq 1} \prod_{l=1}^{N} \frac{1}{d_{i}^{2}} \leq 2\left(\frac{\pi^{2}}{6}\right)^{N}\|g\|_{\infty} .
\end{aligned}
$$

In addition $g * F_{N}$ is a coboundary with respect to convolution by $\mu_{N}$ :

$$
g * F_{N}=\left(\delta_{0}-\mu_{N}\right) * F_{N-1} * g=g * F_{N-1}-\mu_{N} * g * F_{N-1} .
$$

Hence, for all $J$

$$
\left\|\sum_{j=0}^{J} \mu_{N}^{j} * g * F_{N}\right\|_{\infty} \leq 2\left\|g * F_{N-1}\right\|_{\infty} \leq 2^{N}\|g\|_{\infty},
$$

where $\mu_{N}^{j}$ denotes the $j$-fold convolution of $\mu_{N}$ with itself.

By Lemma 2.2, convolution by $\mu_{N}$ is a uniquely ergodic Markov operator whose invariant measure has full support. Hence Theorem 1.1 applies and we may conclude that $g * F_{N}=h-\mu_{N} * h$ for some continuous $h$. By the uniqueness of Fourier coefficients, $h=g * F_{N-1}$. Hence $g * F_{N-1}$ is continuous. It in turn has a bounded cocycle with respect to the measure $\mu_{N-1}$. The same argument shows that $g * F_{N-2}$ is continuous. An iteration of this argument $N-2$ more times shows that $g$ itself is continuous.

Actually a much stronger theorem is true. Its proof, though logically independent, uses closely related ideas.

2.3. Theorem. Let $S$ be a Sidon set and $M$ be a finitely generated multiplicative semigroup. Then $R=S M$ is a Rosenthal set.

Proof. First we require the definition of the height of a function with respect to the essential supremum and some elementary lemmas.

2.4. Definition. Given a real-valued function $p \in \mathbf{L}^{\infty}(\mathbf{T})$ define the height of $p$ with respect to the essential supremum by $p^{\#}(x)=\lim _{\varepsilon \rightarrow 0} \operatorname{ess}_{\sup _{y \in(x-\varepsilon, x+\varepsilon)}} p(y)$. 
2.5. Remark. Given a bounded measurable function $p$, one can find a function $q$ equal to $p$ almost everywhere such that the upper-semicontinuous cover of $q$ of section 1 is equal to the height of $q$ with respect to the essential supremum. Namely, let $q(x)=\operatorname{minimum}\left\{p(x), \inf _{I}\left\|\left.p\right|_{I}\right\|_{\text {ess sup }}\right\}$, where the infimum is taken over all intervals $I$ that contain $x$. However, it is simpler to ignore this fact and establish the relevant properties of the height of a function independently.

2.6. Lemma. For any real-valued $f \in \mathbf{L}^{\infty}(\mathbf{T}), f^{\#}$ is an upper-semicontinuous function.

Proof. Suppose $f^{\#}(x)<\alpha$, for some $\alpha \in \mathbf{R}$. Then there exists an $\varepsilon_{0}$ such that for all $\varepsilon \leq \varepsilon_{0}$, $\operatorname{ess}_{\sup _{y \in(x-\varepsilon, x+\varepsilon)}} f(y)<\alpha$. This implies that $f^{\#}(y)<\alpha$ for all $y \in\left(x-\varepsilon_{0}, x+\varepsilon_{0}\right)$, which is a sufficient condition for upper-semicontinuity.

2.7. Lemma. If $f \in \mathbf{L}^{\infty}(\mathbf{T})$ and $g \in \mathbf{L}^{\infty}(\mathbf{T})$ are real-valued, and $f-g$ is equivalent to a continuous function, then $f^{\#}-g^{\#} \equiv(f-g)^{\#} \equiv f-g$.

Proof. If $F \equiv G$, then $F^{\#}=G^{\#}$ simply as a consequence of the definition of the height of a function. Also from the definition one infers that if $F$ is equivalent to a continuous function, then $F^{\#} \equiv F$, which is the second equivalence of the lemma. One infers the first statement from the following pair of inequalities:

$$
f^{\#}-g^{\#} \leq(f-g)^{\#} \equiv f-g
$$

and

$$
g^{\#}-f^{\#} \leq(g-f)^{\#} \equiv g-f .
$$

2.8. Lemma. If $\mu$ is a positive discrete measure and $f \in \mathbf{L}^{\infty}(\mathbf{T})$ is real-valued, then $\mu * f^{\#} \geq(\mu * f)^{\#}$.

Proof. This is an application of the triangle inequality. We omit the details.

2.9. Lemma. Fix $q$ with $1 \leq q<\infty, f \in \mathbf{L}^{q}(\mathbf{T})$, and a sequence, $\left(M_{j}\right)$, of natural numbers tending to infinity. Then $T_{j} f(x)=\frac{1}{M_{j}} \sum_{m=0}^{M_{j}-1} f\left(x+\frac{m}{M_{j}}\right)$ converges in the $\mathbf{L}^{q}$ norm to the integral of $f$.

Proof. If $f$ is Riemann integrable the conclusion follows from the fact that $T_{j} f(x)$ is a Riemann sum for $f$. On the other hand, for all $j, T_{j}$ is a norm one operator from $\mathbf{L}^{q}(\mathbf{T})$ to $\mathbf{L}^{q}(\mathbf{T})$. If $f$ is arbitrary, then the conclusion follows from approximating $f$ in the $\mathbf{L}^{q}$ norm by continuous functions.

We now begin the proof of Theorem 2.3. Since the finite union of Sidon sets is Sidon (see [LR]), we may suppose without loss of generality that $S$ is symmetric. We prove the theorem by induction on the number of generators of $M$. First suppose that $M$ is generated by a single element. Denote this element by $p$. For $j=0,1,2,3, \ldots$ define $\mu_{j}=\frac{1}{p^{j}} \sum_{k=0}^{p^{j}-1} \delta_{\frac{k}{p^{j}}}$. For $j=0,1,2,3, \ldots \mu_{j}$ is a measure whose transform is two-valued: $\hat{\mu}(k)=1$ if $p^{j}$ divides $k$ and 0 otherwise. Suppose $f \in \mathbf{L}_{S}^{\infty}$. Since $S$ is symmetric, the real and imaginary parts of $f$ both have spectrum in $S$. Hence we may consider them separately and suppose without loss of generality that $f$ is real-valued. Fix $j$. Note that $\left(\delta_{0}-\mu_{j}\right) * f$ is a function with spectrum in a possibly proper subset of $S \cup p S \cup \cdots \cup p^{j-1} S$. This finite union is a 
Sidon set. Hence $\left(\delta_{0}-\mu_{j}\right) * f$ is equivalent to a continuous function. Furthermore Lemmas 2.7 and 2.8 imply that:

$$
\left(\delta_{0}-\mu_{j}\right) * f^{\#} \leq f^{\#}-\left(\mu_{j} * f\right)^{\#} \equiv\left(\left(\delta_{0}-\mu_{j}\right) * f\right)^{\#} \equiv\left(\delta_{0}-\mu_{j}\right) * f .
$$

Since in addition

$$
0=\int\left(\delta_{0}-\mu_{j}\right) * f^{\#} \quad \text { and } \quad\left(\delta_{0}-\mu_{j}\right) * f d m=0,
$$

we may infer that $\left(\delta_{0}-\mu_{j}\right) * f^{\#} \equiv\left(\delta_{0}-\mu_{j}\right) * f$. Now let $j$ tend to infinity. By Lemma 2.9, $f^{\#}-\int f^{\#} d m \equiv f-\int f d m$. This implies that $f$ is equivalent to an upper-semicontinuous function. Replacing $f$ by $-f$ and repeating the argument one infers that $-f$ is also equivalent to an upper-semicontinuous function. Therefore $f$ is equivalent to a lower-semicontinuous function as well. Hence by Lemma 1.5, $f$ is equivalent to a Riemann integrable function. Denote by $\tilde{f}$ a Riemann integrable representative from the equivalence class of $f$. From the definition of the Riemann integral it follows that $\mu_{j} * \tilde{f}$ converges uniformly to the integral of $\tilde{f}$ as $j$ tends to infinity. Hence $\left(\delta_{0}-\mu_{j}\right) * \tilde{f}$ is a Cauchy sequence in the uniform norm. Since each element of the sequence is equivalent to a continuous function, the limit function, $\tilde{f}-\int \tilde{f} d m$, is equivalent to a continuous function. Hence $\tilde{f}$ is equivalent to a continuous function.

The argument is complete in the event that $M$ is generated by a single element. Assume as an induction hypothesis that the theorem is true for finitely generated semigroups with fewer than $m$ generators. Assume that $M$ has $m$ generators. Then $M=M_{1} M_{2}$ where $M_{1}$ is generated by a single element, and $M_{2}$ is generated by $m-1$ elements. Let the generator of $M_{1}$ be denoted by $p$, and as before let $\mu_{j}=\frac{1}{p^{j}} \sum_{k=0}^{p^{j}-1} \delta_{\frac{k}{p^{j}}}$ for $j=0,1,2, \ldots$ Fix $j$. Note that $\left(\delta_{0}-\mu_{j}\right) * f$ is a function with spectrum in a possibly proper subset of $M_{2} S \cup p M_{2} S \cup \cdots \cup p^{j-1} M_{2} S=$ $M_{2}\left(S \cup p S \cup p^{j-1} S\right)$. The term in parentheses is a finite union of Sidon sets and hence itself a Sidon set. $M_{2}$ has $m-1$ generators. Therefore by the induction hypothesis, $\left(\delta_{0}-\mu_{j}\right) * f$ has spectrum in a Rosenthal set. In particular, $\left(\delta_{0}-\mu_{j}\right) * f$ is equivalent to a continuous function. The rest of the argument proceeds as in the case of a single generator,

$$
\left(\delta_{0}-\mu_{j}\right) * f^{\#} \leq f^{\#}-\left(\mu_{j} * f\right)^{\#} \equiv\left(\left(\delta_{0}-\mu_{j}\right) * f\right)^{\#} \equiv\left(\delta_{0}-\mu_{j}\right) * f
$$

and

$$
\int\left(\delta_{0}-\mu_{j}\right) * f^{\#}-\left(\delta_{0}-\mu_{j}\right) * f d m=0 .
$$

Hence $\left(\delta_{0}-\mu_{j}\right) * f^{\#} \equiv\left(\delta_{0}-\mu_{j}\right) * f$. If we let $j$ tend to infinity we may conclude using the same reasoning as above that $f$ itself is equivalent to a continuous function. Thus the induction is complete and the theorem is proved.

2.10. Remark. If it were known that the union of Rosenthal sets were a Rosenthal set, then the above proof could equally well demonstrate that $R M$ is Rosenthal if $R$ is Rosenthal and $M$ is finitely generated.

After the original work of Rosenthal [Ro], Blei [Bl1, Bl2, Bl3] demonstrated the existence of Rosenthal sets in arbitrary compact abelian groups and also exhibited a new class of Rosenthal sets in the integers. It is the latter result that interests us here. Pigno and Saeki [PS1, PS2], building on Blei's work, produced a class of Rosenthal sets that included Rosenthal's original example as a special case: let $E_{n}=$ 
$\{ \pm 1,2, \ldots, n\}$. Let $\left(a_{n}\right)$ be a sequence of integers. Then $E=\bigcup_{n>0} a_{1} a_{2} \cdots a_{n} E_{n}$ is a Rosenthal set, and so is any finite union of translates of $E$. The following corollary to Theorem 2.3 would also suffice to show this result.

2.11. Corollary. Suppose $\left(R_{j}\right)_{j=1}^{\infty}$ is a family of symmetric finite sets and $\left(a_{j}\right)$ is a sequence of integers that satisfies the condition: $a_{j} \mid a_{j+1}$. Then $R=\bigcup_{j>0} a_{j} R_{j}$ is a Rosenthal set. Also, any finite union of translates of $R$ is a Rosenthal set.

Proof. For $j=0,1,2,3, \ldots$ define $\mu_{j}=\frac{1}{a_{j}} \sum_{k=0}^{a_{j}-1} \delta_{\frac{k}{a_{j}}}$. Let $f \in \mathbf{L}_{R}^{\infty}$. Since the $R_{j}$ are symmetric, without loss of generality $f$ is real-valued. We observe that $\left(\delta_{0}-\mu_{j}\right) * f$ is a function with spectrum in $\bigcup_{k=1}^{j-1} a_{k} R_{k}$. The union is a finite set. Thus once again $\left(\delta_{0}-\mu_{j}\right) * f^{\#} \equiv\left(\delta_{0}-\mu_{j}\right) * f$, and letting $j$ tend to infinity the argument proceeds as above to the conclusion that $f$ is equivalent to a continuous function. Thus $R$ is a Rosenthal set.

Suppose $F$ is a finite set of integers. We show that $F+R$ is Rosenthal. If $F$ has one element the statement is true since a set is Rosenthal if and only if every translate of the set is Rosenthal. Assume for the purposes of induction that the statement is true whenever $F$ has fewer than $n$ elements. Suppose $F=\left\{b_{1}, b_{2}, \ldots, b_{n}\right\}$ has $n$ elements. By translating if necessary, we may assume without loss of generality that $0=b_{1}$. Let $f \in \mathbf{L}_{R+F}^{\infty}$. Quite analogously to what has gone before, we observe that $\left(\delta_{0}-\mu_{j}\right) * f$ is a function with spectrum in $T=\bigcup_{k=1}^{j-1} a_{k} R_{k} \bigcup_{k=2}^{n} R+f_{k}$. T is the union of a finite set with a set that is known to be Rosenthal by the inductive hypothesis. Hence $T$ is Rosenthal. Thus once again $\left(\delta_{0}-\mu_{j}\right) * f^{\#} \equiv\left(\delta_{0}-\mu_{j}\right) * f$, and letting $j$ tend to infinity the argument proceeds as above to the conclusion that $f$ is equivalent to a continuous function. Thus $R+F$ is a Rosenthal set.

\section{ACKNOWLEDGEMENTS}

This paper is part of a Ph.D. thesis prepared under the guidance of J. M. Rosenblatt. Neither the paper nor the thesis would have been possible without his generous and patient assistance. I would also like to thank Henry Helson and Kenneth Ross who offered valuable comments on an early version of this paper.

And finally I would like to express an appreciation of my conversations with Randall McCutcheon, Vitaly Bergelson, and Michael Lin. In particular, I initially proved Theorem 1.1 only for convolution operators. Michael Lin and Vitaly Bergelson observed that essentially the same proof carried over to the more general operators considered here, and many aspects of the present exposition were improved by Michael Lin's suggestions.

\section{REFERENCES}

[Bl1] R. C. Blei, on trigonometric series associated with separable, translation invariant subspaces of $\mathbf{L}^{\infty}(G)$, Trans. Amer. Math. Soc. 173 (1972), 491-499. MR 47:2269

[B12] - A simple diophantine condition in harmonic analysis, Studia Math. 52 (1974), 195-202. MR 57:10368

[Bl3] Rosenthal sets that cannot be sup-norm partitioned and an application to tensor products, Colloq. Math. 37 (1977), 295-298. MR 58:2025

[FIS] S. H. Friedberg, A. J. Insel, and L. E. Spence, Linear algebra, Prentice Hall, 1979. MR 80i: 15001

[Fo] S. R. Foguel, Ergodic decomposition of a topological space, Israel J. Math. 7 (1969), 164-167. MR 40:2815

[GH] W. H. Gottschalk and G. A. Hedlund, Topological Dynamics, Amer. Math. Soc. Colloq. Publ., vol. 36, 1955, pp. 135-136. MR 17:650e 
[Kr] Ulrich Krengel, Ergodic theorems, Walter de Gruyter, 1985. MR 87i:28001

[LS] M. Lin and R. Sine, Ergodic theory and the functional equation $(I-T) x=y$, J. Operator Theory 10 (1983), 153-166. MR 84m:47015

[LR] J. M. Lopez and K. A. Ross, Sidon sets, Marcel Dekker, New York, 1975. MR 55:13173

[Me] P. A. Meyer, Probability and potentials, Blaisdell, Waltham, MA, 1966. MR 34:5119

[PS1] L. Pigno and S. Saeki, On the spectra of almost periodic functions, Indiana Univ. Math. J. 25 (1976), 191-194. MR 53:11312

[PS2] _ Almost periodic functions and certain lacunary sets, Kansas State University Technical Report 42, 1974.

[Ro] H. P. Rosenthal, On trigonometric series associated with weak ${ }^{*}$ closed subspaces of continuous functions, J. Math. Mech. 17 (1967), 485-490. MR 35:7064

[St] Stromberg, An introduction to classical real analysis, Wadsworth International Group, 1981. MR 82c:26002

Department of Mathematics, The Ohio State University, Columbus, Ohio 43210

E-mail address: schwartz@math.ohio-state.edu 